\title{
Optically Powered WDM Signal Transmission System With Distributed Parametric Amplification
}

\author{
Xing Xu, Student Member, IEEE, Kim K. Y. Cheung, Sigang Yang, Yu Liang, T. I. Yuk, and \\ Kenneth K. Y. Wong, Member, IEEE
}

\begin{abstract}
We report, for the first time to our knowledge, an optically powered wavelength-division-multiplexing (WDM) communication system with distributed parametric amplification in the dispersion-shifted fiber. The parametric pump for the amplification of WDM signals also acts as the power supply for the receiver component. The proposed scheme is also a potential candidate for the "last mile" transmission. Successful musical signal transmission has been demonstrated. Additionally, four channels of $10-\mathrm{Gb} / \mathrm{s}$ WDM signals are used to get more precise performance evaluation of the system. The power penalties of less than or equal to $1.35 \mathrm{~dB}$ at a bit-error rate of $10^{-9}$ are achieved for all WDM channels in the presence of 10-dB parametric gain.
\end{abstract}

Index Terms-Distributed amplification, fiber-optics communications, optical parametric amplifier (OPA), photovoltaic, power over fiber.

\section{INTRODUCTION}

D ISTRIBUTED parametric amplification (DPA), one of the candidates for the distributed amplification of optical signal, has been studied in recent research [1]. Different from the discrete optical parametric amplifier (OPA) in the specially fabricated highly nonlinear dispersion-shifted fiber (HNL-DSF) [2], [3], DPA adopts the widely available DSF in which the transmission and amplification of the optical signal can be fulfilled simultaneously. The most favorable merit of DPA lies in its capability of minimizing the signal power variation along the transmission span which provides the best tradeoff between the noise figure and fiber nonlinearities [4], [5]. Furthermore, facing the increasing worldwide energy-awareness, in order to enhance the energy-efficiency of the communication system, the residual power of the optical parametric pump can be utilized as the power supply for the receiving component. Actually, optically supplied energy has been well known for a long time, from using solar radiation as a power source for satellites to the more down to earth application example of solar battery for household equipment. In an optically powered communication system, both the optical signal and the power for remote equipment are distributed in the optical fiber.

Manuscript received February 12, 2010; revised April 20, 2010; accepted June 05, 2010. Date of current version July 23, 2010. This work was supported in part by a grant from the Research Grants Council of the Hong Kong Special Administrative Region, China (Project HKU 7179/08E and Project HKU 7183/09E)

The authors are with the Massachusetts Institute of Technology, Cambridge, MA 02139 USA, on leave from the Department of Electrical and Electronic Engineering, The University of Hong Kong, Hong Kong, S.A.R. China (e-mail: xuxing@eee.hku.hk; cheungky@eee.hku.hk; sgyang@eee.hku.hk; yliang@eee.hku.hk; tiyuk@eee.hku.hk; kywong@eee.hku.hk).

Color versions of one or more of the figures in this letter are available online at http://ieeexplore.ieee.org.

Digital Object Identifier 10.1109/LPT.2010.2052796
When we take the "last mile" transmission into account, optically powered fiber link could easily find its position. Unlike the traditional "last mile" transmission, i.e., twisted pairs or electrical cable connections, optical fiber has the advantages of low attenuation, large transmission bandwidth, and high signal-tonoise ratio (SNR), not to mention the economical factor in the long-run of maintenance and repair [6], [7]. Another possible choice for the "last mile" transmission is free space optics (FSO) or optical wireless links [8], [9]. However, in most realistic "last mile" transmission environments, reflection, refraction, or even absorption due to obstacles or unfavorable air conditions like rain and fog, particularly over longer transmission spans, can seriously influence the connections. Recently, with the advances of low-cost high-power lasers, low-power-consumption electronic circuits and highly efficient photodiodes, optically powered communication systems have attracted wide-spread attention. Optically powered radio-over-fiber remote units [10], optically powered LAN systems [11], and optically powered video link [12] have all been demonstrated. The above experiments truly provide potential possibility for the "last mile" transmission, however, these former experiments were multimode-fiber (MMF)-based [10]-[12]. Although MMF could tolerate higher optical power due to larger core dimension, in practice, singlemode fiber (SMF) and DSF could afford much higher data rate and have lower attenuation. More importantly, with the assistance of DPA, the SNR of the transmitted data will be secured for the "last mile" transmission.

While in this letter, together with the demonstration of an optically powered musical signal transmission link, a four-channel optically powered wavelength-division-multiplexing (WDM) signal transmission system with DPA has been achieved within a $10-\mathrm{km}$ DSF. In the mean time, the energy-efficiency of the proposed communication system is guaranteed by a commercially available InP-based concentrated photovoltaic (CPV) cell, which can provide up to $26 \%$ power conversion ratio at the input optical illumination ranged from 1300 to $1550 \mathrm{~nm}$.

\section{PRINCIPLE AND EXPERIMENTAL SETUP}

A simple graph in Fig. 1 shows the scheme of musical signal transmission. The analog musical signal from the MP3 player was first converted to a digital signal string by a digital signal generator (HP 8648C), after which the generated electrical signal was then modulated through a Mach-Zehnder modulator (MZM) onto the optical carrier channel for transmission. The large optical power from the pump branch acted as both the power supply for the remote signal receiver unit and the parametric pump for the signal propagating along a spool of $10-\mathrm{km}$ DSF. The fiber has the loss of $0.20 \mathrm{~dB} / \mathrm{km}$ for both the signal wavelength at around $1543 \mathrm{~nm}$ and the pump wavelength at 


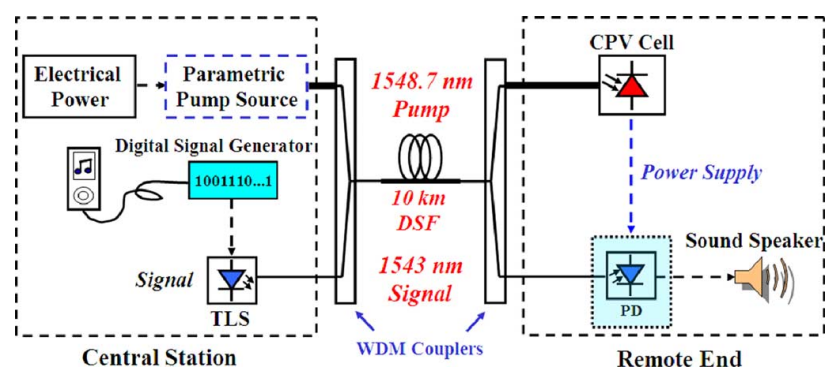

Fig. 1. Optically powered musical signal transmission with DPA (N.B. Some devices used during the experiment have been omitted in the diagram for brevity). CW: continuous wave; TLS: tunable laser source; PD: photodiode; CPV: concentrated photovoltaic cell.

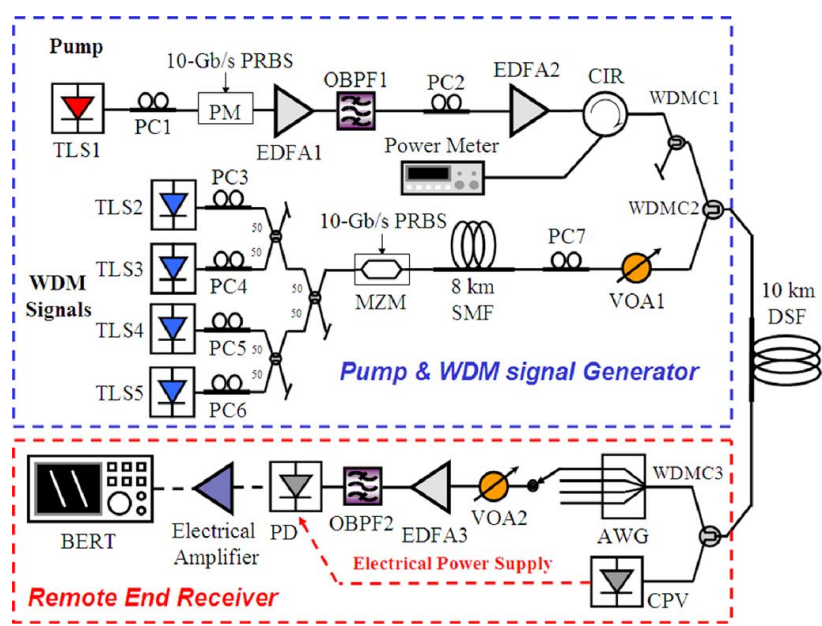

Fig. 2. Experimental setup. WDMC: WDM coupler; AWG: arrayed-waveguide grating.

$1548.8 \mathrm{~nm}$. A 23-dBm pump power at fiber input was adopted to generate a $10-\mathrm{dB}$ gain at the signal wavelength. At the receiver, after convertion by an photodiode (PD) powered by the CPV cell, the signal was then converted back into a digital signal and the clear music was broadcast out by a sound speaker inside the RF communication test set (HP 8920A) used during our experiment at the receiving end.

In order to make practical performance evaluation, a fourchannel WDM signal scheme was introduced. Fig. 2 shows the experimental setup of the transmission system with DPA. The input signals, located at the wavelength of 1542.9, 1543.76, 1544.65 , and $1545.45 \mathrm{~nm}$, respectively, were generated by combining the output of four continuous-wave $(\mathrm{CW})$ tunable laser sources (TLS2-5) with three 50/50 couplers and then intensitymodulating them together by an MZM which was driven by a $10-\mathrm{Gb} / \mathrm{s} 2^{7}-1$ pseudorandom binary sequence (PRBS) from the bit pattern generator (BPG). The polarization controllers (PC3-6) after each laser source were used to minimize the insertion loss of each channel by aligning its state of polarization (SOP) with the transmission axis of the MZM. A spool of 8-km SMF was deployed to decorrelate different channels in the time domain. On the other branch, the parametric pump, sourced from TLS1 wavelength at $1548.8 \mathrm{~nm}$, was first phase-dithered with a phase modulator (PM) which was driven by a $10-\mathrm{Gb} / \mathrm{s}$ $2^{23}-1$ PRBS, to suppress the stimulated Brillouin scattering (SBS). A double-stage erbium-doped fiber amplifier (EDFA) scheme was applied to amplify the pump power upto $23 \mathrm{dBm}$

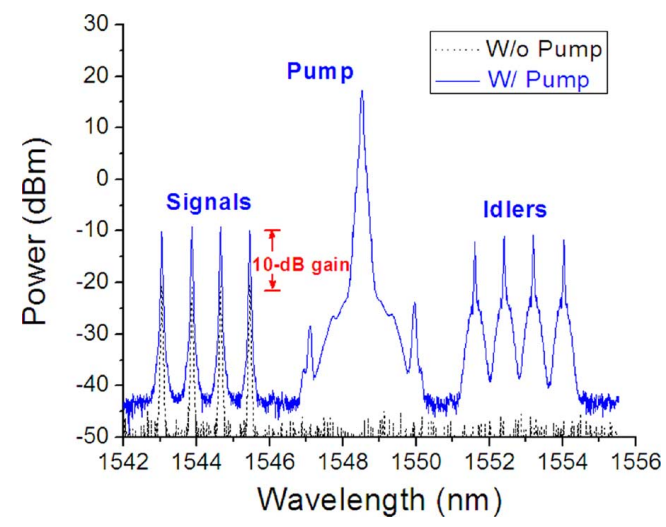

Fig. 3. Optical spectra at the output of DSF with (blue solid line) and without (black dotted line) pump. (OSA resolution bandwidth: $0.02 \mathrm{~nm}$ ).

before the fiber input. The optical bandpass filter (OBPF1) between two EDFAs was used to reduce the amplified spontaneous emission (ASE) noise level from EDFA1. A circulator (CIR) was inserted after EDFA2 to avoid the reflection and monitor the SBS level. One WDM coupler (WDMC1) with the passing band of $3 \mathrm{~nm}$ around $1549 \mathrm{~nm}$ was applied to trim down the ASE noise from EDFA2. After combining with WDMC2, the pump and the signals were then launched together into the 10-km DSF which has the nonlinear coefficient $\gamma$ of $2 \mathrm{~W}^{-1} \cdot \mathrm{km}^{-1}$ and the zero-dispersion wavelength (ZDW) $\lambda_{0}$ of $1548 \mathrm{~nm}$. By controlling the variable optical attenuator (VOA), the signal powers at the input of DSF were maintained at less than $-19 \mathrm{dBm}$, while the pump power at the same point was $19.5 \mathrm{dBm}$. This ensured that the pump was not depleted and the generation of signal-related nonlinear effects, such as four-wave-mixing (FWM) and cross-gain modulation (XGM), would be insignificant. The SOP of the pump and signals were aligned by PC2 and PC7 to attain the maximum optical parametric gain.

The optical spectra at the output of DSF with and without pump are shown in Fig. 3. The power of four channels at the input of DSF was $-19.3,-18.92,-19.16$, and $-19.07 \mathrm{dBm}$, respectively. The corresponding ON-OFF gains were 10.06, $10.57,10.83$, and $10.33 \mathrm{~dB}$. At the receiving end, after WDMC3, the residual pump power of $15.2 \mathrm{dBm}(33.1 \mathrm{~mW})$ was sent to the CPV cell to generate the electrical power for the signal receiving component. Finally, four channels of $10-\mathrm{Gb} / \mathrm{s}$ WDM nonreturn-to-zero (NRZ) signals were demultiplexed by an arrayed-waveguide grating (AWG) and the performance of each channel was then quantified by the bit-error-rate tester (BERT).

\section{EXPERIMENTAL RESULTS AND DISCUSSION}

Fig. 4 lists the measured eye-diagrams of the received $10-\mathrm{Gb} / \mathrm{s}$ NRZ signals. Clear and widely open eye-diagrams are observed for all channels, which indicate the high quality of the receiving WDM signals. The noise observed on the mark level is mainly due to the conversion of pump phase modulation to signal intensity modulation along the DSF with the process of parametric amplification.

Each channel was turned OFF sequentially to quantify the signal crosstalk amongst channels as shown in Fig. 5. It can be observed that there is no observable spurious FWM components 


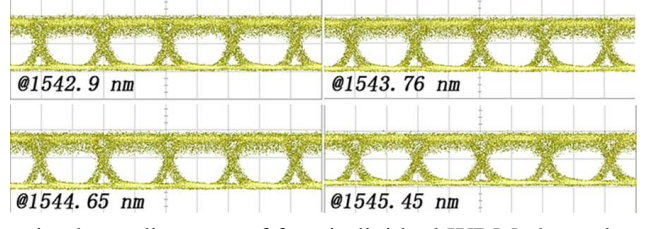

Fig. 4. Received eye-diagrams of four individual WDM channels.
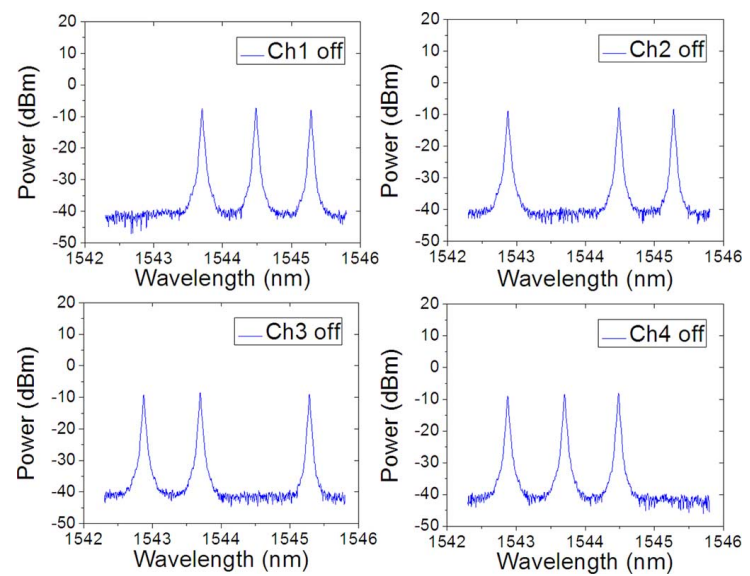

Fig. 5. Spectra with one channel OFF after DPA. (OSA resolution bandwidth: $0.02 \mathrm{~nm})$.
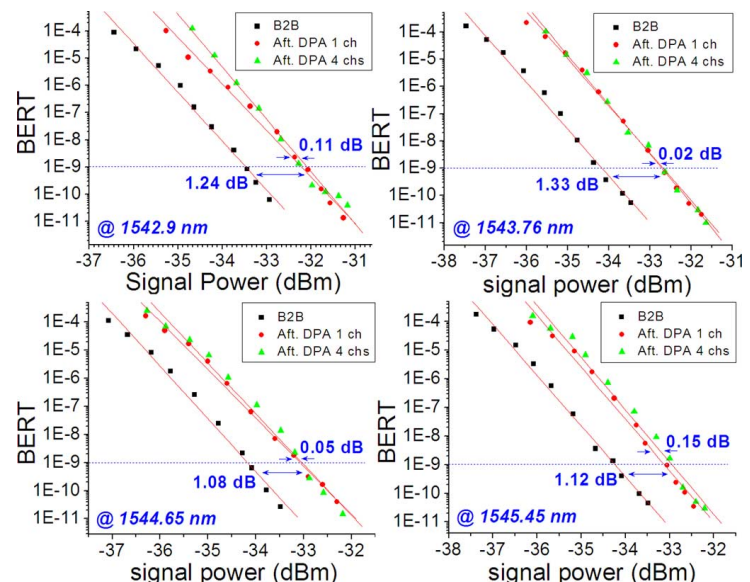

Fig. 6. BER plots for the back-to-back signals and the signals after DPA: only one channel ON ( 1 ch: circle); 4 channels ON (4 chs: triangle).

(more than $28 \mathrm{~dB}$ lower than the signals) generated. The corresponding BER of each channel was measured and compared against the back-to-back (B2B) signals (Fig. 6). Two different configurations were considered during the BER measurement process: 1) only one channel was turned oN; 2) all four channels were turned ON. Error-free operations are attained for all channels and the power penalties ranged from 1.08 to $1.33 \mathrm{~dB}$ at a BER of $10^{-9}$ for the single-channel case; additional penalties from 0.02 to $0.15 \mathrm{~dB}$ were measured in the four-channel case which indicate nearly negligible impact on these WDM channels.

Fig. 7 shows the output curve of the CPV cell with different input optical power at the pump wavelength. According to the curve, the corresponding output voltage could reach $4.53 \mathrm{~V}$, which was enough for the bias voltage of the $5-\mathrm{V}$ photodiode (PD) as the optical signal to electrical signal converter. Considering the relatively small amount of power at the far-end subscriber $(6.6 \mathrm{~mW}$ in our experiment, according to the $20 \%$ typ-

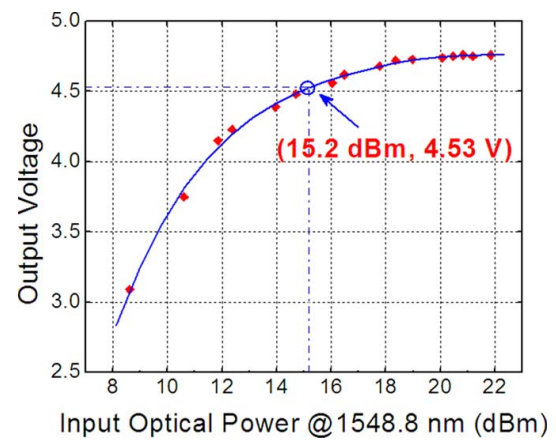

Fig. 7. Response curve of the CPV cell with the input pump light at $1548.8 \mathrm{~nm}$.

ical energy conversion ratio of the CPV cell), in addition to the possible deployment in the "last mile" transmission, potential application lies in the optically powered sensor network located in a relatively inaccessible environment. Our scheme of combining DPA and optical power transmission provides a potential method for the recycle of optical pump in distributed amplification.

\section{CONCLUSION}

We have demonstrated an optically powered WDM signal transmission system with DPA in the DSF. Less than or equal to $1.35-\mathrm{dB}$ power penalties at the BER of $10^{-9}$ are obtained for all channels at the presence of $10-\mathrm{dB}$ parametric gain. The incorporation of DPA and optical power transmission during the experiment provides a new solution for the "last mile" transmission.

\section{REFERENCES}

[1] G. Kalogerakis, M. E. Marhic, K. K. Y. Wong, and L. G. Kazovsky, "Transmission of optical communication signals by distributed parametric amplification," J. Lightw. Technol., vol. 23, no. 10, pp. 2945-2953, Oct. 2005.

[2] M. E. Marhic, N. Kagi, T. K. Chiang, and L. G. Kazovsky, "Broadband fiber optical parametric amplifiers," Opt. Lett., vol. 21, pp. 573-575, 1996.

[3] J. Hansryd, P. A. Andrekson, M. Westlund, J. Li, and P. O. Hedekvist, "Fiber-based optical parametric amplifiers and their applications," IEEE J. Sel. Topics Quantum Electron., vol. 8, no. 3, pp. 506-520, May/Jun. 2002.

[4] C. McKinstrie, M. Yu, M. G. Raymer, and S. Radic, "Quantum noise properties of parametric processes," Opt. Express, vol. 13, no. 13, pp. 4986-5012, 2005.

[5] M. Vasilyev, "Distributed phase-sensitive amplification," Opt. Express, vol. 13, no. 19, pp. 7563-7571, 2005.

[6] D. E. A. Clarke and T. Kanada, "Broadband: The last mile," IEEE Commun. Mag., vol. 31, no. 3, pp. 94-100, Mar. 1993.

[7] P. E. Green Jr., "Paving the last mile with glass," IEEE Spectr., vol. 39, no. 12 , pp. 13-14, Dec. 2002.

[8] E. Leitgeb, J. Bregenzer, P. Fasser, and M. Gebhart, "Free space optics-Extension to fiber-networks for the 'last mile'," in Proc. 15th Annu. Meeting IEEE LEOS, 2002, 2002, vol. 2, pp. 459-460.

[9] E. Leitgeb, M. Loschnigg, U. Birnbacher, G. Schwarz, and A. Merdonig, "High reliable optical wireless links for the last mile access," in Proc. 10th Anniversary Int. Conf. Transparent Optical Networks, 2008, 2008, vol. 4, pp. 178-183.

[10] D. Wake, A. Nkansah, N. J. Gomes, C. Lethien, C. Sion, and J. P. Vilcot, "Optically powered remote units for radio-over-fiber systems," J. Lightw. Technol., vol. 26, no. 15, pp. 2484-2491, Aug. 1, 2008.

[11] H. Miyakawa, Y. Tanaka, and T. Kurokawa, "Design approaches to power-over-optical local-area-network systems," Appl. Opt., vol. 43, pp. 1379-1389, 2004.

[12] G. Bottger, M. Dreschmann, C. Klamouris, M. Hubner, M. Rger, A. W. Bett, T. Kueng, J. Becker, W. Freude, and J. Leuthold, "An optically powered video camera link," IEEE Photon. Technol. Lett., vol. 20, no. 1, pp. 39-41, Jan. 1, 2008. 